\title{
Activation of the Nrf2-ARE Pathway Ameliorates Hyperglycemia-Mediated Mitochondrial Dysfunction in Podocytes Partly Through Sirt1
}

\author{
Qunzi Zhang $^{a} \quad$ Qiongxia Deng $^{b} \quad$ Jun Zhang ${ }^{b} \quad$ JianTing Ke $^{\mathrm{a}} \quad \mathrm{Ye} \mathrm{Zhu}^{\mathrm{a}}$ \\ Ruo wei Wen $^{\mathrm{b}}$ Zengchun Ye ${ }^{\mathrm{b}}$ Hui Peng ${ }^{\mathrm{b}}$ Zhong zhen Su${ }^{\mathrm{c}}$ Cheng Wang ${ }^{\mathrm{a}}$ \\ Tanqi Loub \\ aDivision of Nephrology, Department of Medicine, the $5^{\text {th }}$ Hospital of Sun Yat-sen University, Zhuhai, \\ Guangdong, bDivision of Nephrology, Department of Medicine, the $3^{\text {rd }}$ Hospital of Sun Yat-sen \\ University, Guangzhou, Guangdong, 'Department of Ultrasound, the $5^{\text {th }}$ Hospital of Sun Yat-sen \\ University, Zhuhai, Guangdong, China
}

\section{Key Words}

Antioxidant response element - Diabetes mellitus - Podocytes - Tert-butylhydroquinone • Sirt1

\begin{abstract}
Background/Aims: Previously we have shown that activation of the nuclear factor (erythroidderived 2)-like 2 ( $\mathrm{Nrf2}$ )-antioxidant response element (ARE) attenuated hyperglycemiainduced damage in podocytes, but the molecular mechanism remains unknown. Methods: Tert-butylhydroquinone (t-BHQ) and small interfering RNAs (siRNAs) were used to regulate Nrf2 expression, while nicotinamide and siRNAs were used to regulate sirtuin 1 (Sirt1) activity and expression, respectively. Mitochondrial superoxide, membrane potential and ATP levels were measured to assess changes in mitochondrial function. Nephrin and synaptopodin expression were measured by western blot analysis. Human podocytes and $\mathrm{db} / \mathrm{db}$ diabetic mice were used in this study. Results: t-BHQ pretreatment of human podocytes exposed to high glucose $(\mathrm{HG})$ alleviated mitochondrial dysfunction, enhanced the expression of Sirt1, nephrin and synaptopodin and lowered BSA permeability compared with podocytes exposed to HG without t-BHQ pretreatment $(p<0.05)$. Human podocytes exposed to HG had more severe mitochondrial dysfunction, lower expression of Sirt1, synaptopodin and nephrin and higher BSA permeability than podocytes exposed to HG when Nrf2 expression was downregulated by siRNAs $(p<0.05)$. The protection provided by activation of the NrfARE pathway in podocytes exposed to HG was partially diminished when Sirt1 expression or activity was decreased by siRNAs or inhibitor compared with podocytes exposed to HG and pretreated with t-BHQ $(p<0.05)$. When nicotinamide and $\mathrm{t}-\mathrm{BHQ}$ were both administered
\end{abstract}

Qunzi Zhang, Qiongxia Deng and Jun Zhang contributed equally to this work.

Dr Cheng Wang

and Dr Zhongzhen Su
Division of Nephrology, Department of Medicine, the $5^{\text {th }}$ Hospital of Sun Yat-sen

University, Zhuhai, Guangdong, (China)

E-Mailwt770716@163.com; sp9313@126.com 
to $\mathrm{db} / \mathrm{db}$ mice, we observed higher levels of urinary albumin/creatinine, lower nephrin and synaptopodin expression, more severe mesangial matrix deposition, collagen deposition on pathological slides and mitochondrial structural damage in podocytes compared to $\mathrm{db} / \mathrm{db}$ mice treated only with t-BHQ. Conclusions: Our findings suggest that crosstalk between Sirt1 and the Nrf2-ARE anti-oxidative pathway forms a positive feedback loop and that protection provided by t-BHQ activation of the Nrf2-ARE pathway in $\mathrm{db} / \mathrm{db}$ mice is partly dependent on Sirt1.

(C) 2018 The Author(s)

Published by S. Karger AG, Basel

\section{Introduction}

Diabetic Kidney Disease (DKD) is the main cause of end-stage renal disease [1-2]. It has been established that hyperglycemia plays a crucial role in the pathogenesis of DKD. High glucose (HG) levels induce metabolic abnormalities in several glucose metabolic pathways and induce mitochondrial dysfunction, with subsequent overproduction of reactive oxygen species (ROS) which may in turn contribute to the development of the various microvascular and macrovascular pathologies observed in diabetes [3-5].

Podocytes are highly differentiated epithelial cells located in the glomerular basement membrane which form the outermost layers of glomerular filtration, and injuries to these cells play an important role in the progression of diabetic nephropathy [6,7]. DKD rat models have shown that early glomerular changes involve podocyte injury without mesangial expansion [8], and a correlation between the rate of albumin excretion and a drop in podocyte numbers has been found in patients with DKD $[9,10]$. The progression of diabetic nephropathy in both type-1 and type-2 diabetes has been associated with podocyte loss [11,12].

Mitochondria are complex intracellular organelles that are responsible for various metabolic functions, includingenergy production via oxidative phosphorylation. Mitochondria are also a major source of ROS, and the overproduction of ROS damages mitochondrial DNA and the oxidation respiratory chain, which ultimately causes mitochondrial dysfunction: increased ROS production, the accumulation of impaired mitochondrial DNA and progressive respiratory chain dysfunction [13-15]. Mitochondrial dysfunction is an early event in aldosterone-induced podocyte injury [16], and maintaining normal mitochondrial function by genetic methods protects against podocyte depletion and phenotypic changes $[17,18]$.

Nuclear factor (erythroid-derived 2)-like 2 (Nrf2) plays an important role in defense against oxidative stresses [19]. It induces the expression of antioxidant genes that protect many cell types from oxidative stress caused by tissue damage resulting from inflammation and injury. Previously, we have showed that activation of the Nrf2-antioxidant response element (ARE) pathway protect against mouse podocyte damage induced by HG [20], but the underlying mechanism remains unknown.

Sirtuin 1 (Sirt1), an $\mathrm{NAD}^{+}$-dependent protein deacetylase, plays multiple roles in cells involving stress resistance, DNA repair, cell senescence, inflammation and mitochondrial regulation [21]. As a pivotal protein in cellular metabolism, the regulatory effect of Sirt1 on mitochondrial dynamics has been established [22]. Huang et al. have demonstrated that crosstalk between Sirt1 and the Keap1/Nrf2/ARE anti-oxidative pathway forms a positive feedback loop to inhibit protein expression of fibronectin and transforming growth factor- $\beta 1$ in advanced glycation-end products-treated in rat glomerular mesangial cells treated with advanced glycation end products [23]. However, it remains to be determined if Sirt1 plays a role in activation of the Nrf2-ARE pathway. Hence, we hypothesized that Sirt1 is involved in activation of this pathway and ameliorates hyperglycemia-mediated mitochondrial dysfunction in podocytes.

\section{Materials and Methods}

Chemicals and Reagents

Tert-Butylhydroquinone (t-BHQ) (112941) and Nicotinamide (72340) were purchased from SigmaAldrich (St. Louis, MO, USA). The specific Nrf2 and SIRT1 small-interfering RNA (SiRNA), negative control of 


\section{Cellular Physiology Cell Physiol Biochem 2018;48:1-15 \\ \begin{tabular}{l|l} 
and Biochemistry Published $10.1159 / 000491658$ & $\begin{array}{l}\text { C } 2018 \text { The Author(s). Published by S. Karger AG, Basel } \\
\text { www.karger.com/cpb }\end{array}$
\end{tabular} \\ Zhang et al.: Nrf2-ARE Pathway in Mitochondrial Dysfunction}

SiRNA were purchased from Ribobio company(Guangzhou,China), Lipofectamine ${ }^{\mathrm{TM}} 3000$ Reagent, MitoSOX ${ }^{\mathrm{TM}}$ red mitochondrial superoxide indicator and JC-1 were purchased from Invitrogen (Carlsbad,CA,USA).

\section{Cell culture}

Human podocytes were kindly provided by Prof. Saleem and maintained in RPMI 1640 medium supplemented with 10\% fetal bovine serum (FBS; Sigma-Aldrich), Insulin-Transferrin-Selenium supplement (ITS; Sigma-Aldrich), $2 \mathrm{mM}$ L-glutamine and antibiotic mixture, as previously reported [24]. To stimulate cell proliferation, podocytes were cultivated at $33^{\circ} \mathrm{C}$ in $5 \% \mathrm{CO}_{2}$ (permissive conditions). To induce differentiation, they were maintained at $37^{\circ} \mathrm{C}$ in $5 \% \mathrm{CO}_{2}$ (non-permissive conditions) for at least 2 weeks.

Growth-arrested podocytes were incubated with normal glucose (Control, 5mmol/l) and high glucose (HG,30mmol/l) medium for 72 hours, respectly. The widely used Nrf2 activator, t-BHQ(TertButylhydroquinone,20umol/l) was pretreated for 4 hours to investigate the potential role of Nrf2-ARE pathway. The classical SIRT1 antagonist, nicotinamide $(20 \mathrm{mmol} / \mathrm{l})$ was pretreated for 2 hours to study the potential role of SIRT1 in human podocyte incubated with HG. All doses and time of these treatments were determined in our pilot studies.

\section{SiRNA transfection}

Transient transfection of siRNA was carried out according to Santa Cruz's protocol as described previously [19]. The special sequences of Sirt1-siRNA were: sense:5'-CCAGUAGCACUAAUUCCAATT-3', antisense: 5'-UUGGAAUUAGUGCCACUGGTT-3'. The special sequences of Nrf2-siRNA were: sense: 5'-GAGGAUGGGAAACCUUACUTT3', antisense: 5'-AGUAAGGUUUCCCAUCCUCTT-3'. For each transfection, 100ul transfection medium containing 4ul SiRNA stock solution was gently mixed with 100ul transfecition medium contaning 5 ul transfection reagent. After 25 minutes incubation at $37^{\circ} \mathrm{C}$, complexes were added to the cells in $2 \mathrm{ml}$ transfection medium, and cells were incubated with this mixture for $6 \mathrm{~h}$ at $37^{\circ} \mathrm{C}$. The transfection medium was then replaced with normal medium, and cells were cultured for 48 hours.

\section{BSA permeability assay}

BSA permeability assay was used to evaluate the filtration barrier function of podocyte monolayers. Briefly, podocytes $\left(5^{*} 10^{3}\right)$ were seeded onto collagen-coated trans-well filters ( pore size, 3 um; Corning,New York,NY,USA) and cultured under differentiation conditions. Cells were washed twice with PBS. Then, the top chamber was refilled with $0.2 \mathrm{ml}$ of RPMI 1640 medium and the bottom chamber with $1 \mathrm{ml}$ of RPMI 1640 medium containing $10 \mathrm{ug} / \mathrm{ml}$ FITC-BSA, and incubated at $37^{\circ} \mathrm{C}$. After 4 hours of incubation, a small amount of medium from the top chamber was collected and the fluorescence of FITC-BSA determined.

\section{Living cell imaging}

Mitochondrial superoxide is generated as a byproduct of oxidative phosphorylation. MitoSOX Red mitochondrial superoxide indicator(M36008, Invitrogen,USA) is a novel fluorogenic dye for highly selective detection of superoxide, indicated by a fluorescence emission at $520 \sim 580 \mathrm{~nm}$. Dissolve the contents (50 $\mu \mathrm{g}$ ) of one vial of MitoSOX mitochondrial superoxide indicator in $13 \mu \mathrm{L}$ of dimethylsulfoxide (DMSO) to make a $5 \mathrm{mM}$ MitoSOX ${ }^{\mathrm{TM}}$ reagent stock solution.Dilute the $5 \mathrm{mM}$ MitoSOX reagent stock solution in HBSS buffer(GIBCO,USA) to make a $5 \mu \mathrm{M}$ MitoSOX reagent working solution. Apply $1.5 \mathrm{~mL}$ of $5 \mu \mathrm{M}$ MitoSOX reagent working solution to cover cells adhering to coverslips, then incubate cells for 10 minutes at $37^{\circ} \mathrm{C}$ in dark place, finally wash cells gently three times with warm buffer.

JC-1(5',6, 6'-tetrachloro-1, 1',3, 3'-tetraethylbenzimidazolylcarbocyanineiodide), exhibits potentialdependent accumulation in mitochondria, indicated by a fluorescence emission shift from green $(\sim 529 \mathrm{~nm})$ to red ( $\sim 590 \mathrm{~nm})$. Consequently, mitochondrial depolarization is indicated by a decrease in the red/green fluorescence intensity ratio. The potential-sensitive color shift is due to concentration-dependent formation of red fluorescent J-aggregates._The ratio of green to red fluorescence is dependent only on the membrane potential. Apply $1.5 \mathrm{~mL}$ of $2 \mu \mathrm{M} \mathrm{JC}-1$ reagent working solution to cover cells adhering to coverslips. Incubate cells for $15-20$ minutes at $37^{\circ} \mathrm{C}$, protected from light. Wash cells gently three times with warm buffer.

The nuclei were counterstained with 4', 6-diamidino-2-phenylindole (DAPI) (Antgene, China) for $5 \mathrm{~min}$. All microscopy images were recorded using a confocal microscope (Olympus, Japan).

\section{ARE luciferase reporter gene assay}

The activity of the Nrf2 antioxidant pathway was measured with ARE reporter kit according to manufacturer instructions. Briefly, podocytes were plated in 96-well culture plates 1 day before transfection. 


\section{Cellular Physiology Cell Physiol Biochem 2018;48:1-15 and Biochemistry Published onlune: July $11,2018 \quad \begin{aligned} & \text { DOI: 10.1159/000491658 } 2018 \text { The Author(s). Published by S. Karger AG, Basel } \\ & \text { www.karger.com/cpb }\end{aligned}$ \\ Zhang et al.: Nrf2-ARE Pathway in Mitochondrial Dysfunction}

Cells were then co-transfected with reporter and negative control reporter using Lipofectamine 3000 reagent. These transfected cells were then exposed to their respective treatment. Luciferase activities were measured with the Dual-Glo Luciferase Assay system. The luciferase activities of the firefly were normalized to those of the internal control.

\section{SIRT1 activity assay}

SIRT1 activity was measured using an SIRT1 activity assay kit (ab156065, Abcam,USA). After extracting the nuclear fraction, fluorescence intensity was detected at $340 \mathrm{~nm}$ excitation and $460 \mathrm{~nm}$ emission using a microplate fluorescence reader (Thermo Scientific, USA). Total podocytes proteins were determined using a BCA protein assay kit (P0010, Beyotime Tech, Shanghai, China). SIRT1 activity was normalized to their respective protein concentrations and expressed as the fold change relative to the Control group.

\section{ATP assay}

ATP Assay Kit (S0026, Beyotime Tech, Shanghai, China) that can be used to detect the level of ATP(adenosine 5 'adenosine $5^{\prime}$-triphosphate) in podocytes. Total cultured podocytes were cracked by the addition of lysis buffer at $4{ }^{\circ} \mathrm{C}$. The suspension was centrifuged at $14,000 \times \mathrm{g}$ for 10 minutes, and further detect the concentration of ATP in suspension. For ATP assay, all samples were evaluated using luminometer(Tenon, China).

\section{Animal studies}

Male $\mathrm{db} / \mathrm{db}$ and $\mathrm{db} / \mathrm{m}$ mice (12 weeks old, Nanjing University, Nanjing, China) were maintained on standard chow, housed at $25^{\circ} \mathrm{C}$ and $50-60 \%$ relative humidity, in a $12 \mathrm{~h} / 12 \mathrm{~h}$ light/dark cycle, and allowed free access to tap water. All animal procedures conformed to the Guide for Care and Use of Laboratory Animals by the National Institute of Health for Laboratory Animal Research and were approved by the Sun Yat-sen University Animal Care and Use Committee. $D b / d b$ and $\mathrm{db} / \mathrm{m}$ mice were randomly divided into groups and received the following treatments at 12 weeks: the normal saline group with saline gavage treatment (NS, $n$ = 6), t-BHQ group (t-BHQ, 10\% t-BHQ drinking water) ( $\mathrm{n}=6$ ), Nicotinamide-gavaged group (NICO, 50mg/ $\mathrm{kg} /$ day Nicotinamide by gavage) $(\mathrm{n}=6)$, $\mathrm{t}-\mathrm{BHQ}$ and Nicotinamide-gavaged group ( $\mathrm{t}-\mathrm{BHQ}+\mathrm{NICO}, 50 \mathrm{mg} / \mathrm{kg} /$ day Nicotinamide by gavage and $10 \%$ o t-BHQ drinking water) $(n=6)$. These mice were sacrificed ethically after 12 weeks. Blood samples and kidney cortex tissue were collected and stored $-80^{\circ} \mathrm{C}$.

\section{Western blot analysis}

Total proteins that from podocytes and renal tissues were extracted by the addition of radio immunoprecipitation assay(RIPA) lysis buffer (Beyotime Tech, Shanghai, China) containing 1mM PMSF at 4 ${ }^{\circ} \mathrm{C}$. And tissues were further sonicated using ultrasound. The suspension was centrifuged at $14,000 \times \mathrm{g}$ and the medium containing the proteins was collected. For Western blot analysis, a $10 \%$ sodium dodecyl sulfatepolyacrylamide gel was run under standard conditions by loading $50 \mathrm{ug}$ total proteins in each lane. The gel was transfered to a polyvinylidene fluoride membrane at $100 \mathrm{v}$ for 1 hour 30 minutes. The membrane was rinsed in Tris-buffered saline followed by rinsing in blocking buffer (5\% milk powder) for 5 minutes. The membrane was immersed in blocking buffer for 1 hour before incubation with primary antibodies overnight at $4{ }^{\circ} \mathrm{C}$. The primary antibodies are: Rabbit monoclonal anti-NQ01(ab80588, abCAM,1:1000 dilution); antiSIRT1(9475S, cell signaling tech,1:1000); Rabbit polyclonal anti-Nephrin(ab58968, abCAM,1:250); antiSynaptopodin(ab117702, abCAM,1:250); anti-Nrf2(sc13032,Santa Cruz,1:100); anti- $\beta$-Actin(4970S,cell signaling tech,1:5000). After rinsing in wash buffer, horseradish peroxidase-conjugated secondary antibody was used for 1 hour at room temperature. After the final wash, the membrane was developed using ECL Reagent, and densitometric analyses conducted using ImageJ Software.

\section{Animal biochemical parameter analysis}

The mice were housed in metabolic cages (Nalgene Nunc International, Rochester, NY, USA). Urine was collected over 24 hours and placed into tubes containing antibiotics. Microalbumin was determined with Albuwell M (Exocell, Philadelphia, PA, USA). The values were normalized to creatinine, which was measured using the Creatinine Companion (Exocell, Philadelphia, PA, USA). Fasting blood glucose was measured using the OneTouch UltraSmart Blood Glucose Meter (Lifescan, Milpitas,CA, USA) every 4 weeks. Blood urine nitrogen levels were measured using a blood urea nitrogen enzymatic kit (Bio Scientific, Austin, TX, USA). 
Pathological staining

For the morphometric studies, the kidneys were fixed in 10\% neutral buffered formalin and subsequently embedded in paraffin. Four-micrometer paraffin sections of kidney cortex tissue were dewaxed. hematoxylin was used to stain the nuclei and eosin was used to dye the cytoplasm. The sections were then dehydrated and sealed with neutral balsam. The 4- $\mu$ m sections of the paraffin-embedded tissues were stained with periodic acid-Schiff (PAS) and Masson. Digital images of the glomeruli were obtained using light microscopy (200x). The area of the glomeruli and the mesangial matrix was quantified in a blinded fashion using an image analysis system. The mesangial matrix index was calculated and graded as follows: 0 represents no lesion, $1+$ represents sclerosis of $<25 \%$ of the glomerulus, whereas $2+, 3+$, and $4+$ represent sclerosis of $>25-50,>50-75$, and $>75 \%$ of the glomerulus $[25,26]$.

\section{Statistical analyses}

Data are expressed as mean \pm standard deviation unless stated otherwise. Statistical differences were assessed using two-tailed multivariate ANOVA for repeated measures. And $\mathrm{p}<0.05$ was considered statistically significant.

\section{Results}

HG-induced mitochondrial dysfunction and injuries in human podocytes

Human podocytes incubated in HG showed mitochondrial dysfunction: lower ATP levels, higher superoxide levels detected by mitoSOX and decreased mitochondrial depolarization. Simultaneously, these podocytes showed lower expression of synaptopodin and nephrin and higher BSA permeability compared with podocytes exposed to normal glucose $(p<0.05$; Fig. 1 and 4).

Role of the Nrf2-ARE pathway in protection against mitochondrial dysfunction and injuries to human podocytes induced by $H G$

T-BHQ pretreatment of human podocytes exposed to HG increased Nrf2 transcriptional activity and enhanced total Nrf2 and NAD(P)H quinone dehydrogenase 1 (NQO1) protein, which suggested that $\mathrm{t}-\mathrm{BHQ}$ activated the Nrf2-ARE pathway. In addition, t-BHQ pretreatment also improved mitochondrial dysfunction and podocyte injuries after HG exposure, including increased ATP levels, decreased mitoSOX, improved mitochondrial depolarization, enhanced expression of nephrin and synaptopodin, and decreased BSA-mediated permeability compared to those podocytes which not pretreated with t-BHQ. However, when Nrf2 expression was downregulated by siRNAs, human podocytes exposed to HG had more severe mitochondrial dysfunction and injuries, including lower ATP levels, higher mitoSOX and decreased mitochondrial depolarization, lower expression of synaptopodin and nephrin and higher BSA permeability than podocytes exposed to HG without siRNA treatment $(p<0.05)$. These data all indicated that activation of the Nrf2-ARE pathway could attenuate mitochondrial dysfunction and injuries to human podocytes induced by HG (Fig. 1, 2 and 4).

The effect of the Nrf2-ARE pathway on Sirt1 expression and activity in human podocytes exposed to $H G$

The activity and expression of Sirt1 in human podocytes exposed to HG were decreased when compared with podocytes exposed to normal glucose $(p<0.05)$, but after pretreatment with t-BHQ, the activity and expression of Sirt1 in human podocytes exposed to HG were increased compared with podocytes incubated with HG medium without $\mathrm{t}-\mathrm{BHQ}$ pretreatment $(p<0.05)$. We also downregulated the Nrf2-ARE pathway by siRNAs to explore changes in expression and activity of Sirt1. We found that activity and expression of Sirt1 in Nrf2-silenced human podocytes exposed to HG were decreased compared with podocytes incubated with HG medium without Nrf2 silencing $(p<0.05)$. The data suggested that the Nrf2-ARE pathway may regulate the expression and activity of Sirt1 in human podocytes exposed to HG (Fig. 2). 
The role of Sirt1 in mitochondrial dysfunction and injuries to human podocytes induced by $H G$

To test the role of Sirt1 in mitochondrial dysfunction and injuries to human podocytes induced by HG, we examined the effect of Sirt1 siRNA and Sirt1 activity inhibitor nicotinamide in the following experiments. When we decreased the deacetylase activity and expression of Sirt1 in these podocytes, exposure to HG produced more severe mitochondrial dysfunction and damage: lower ATP levels, higher mitoSOX and decreased mitochondrial depolarization, lower expression of synaptopodin and nephrin and higher BSA permeability compared with podocytes incubated with HG without Sirt1 inhibition $(p<0.05)$. These results indicated that Sirt1 plays a role in protecting against mitochondrial dysfunction and injuries to human podocytes induced by HG (Fig. 3 and 4).

The role of Sirt1 in the Nrf2-ARE pathway of human podocytes induced by HG

Compared with podocytes exposed to HG alone, the expression and transcriptional activity of Nrf2 and NQ01 were decreased in podocytes exposed to HG when Sirt1 was downregulated by siRNAs and inhibitor $(p<0.05)$, which indicated that Sirt1 and the Nrf2ARE pathway may undergo potential interactions in human podocytes exposed to HG (Fig. $3)$.

The role of Sirt1 in the Nrf2-ARE pathway protecting against mitochondrial dysfunction and injuries to human podocytes induced by $H G$

The protection provided by activation of the Nrf2-ARE pathway in podocytes exposed to HG was partially diminished when Sirt1 expression or activity was decreased by siRNAs or inhibitor. Sirt1 inhibition produced lower ATP levels, higher mitoSOX and decreased mitochondrial depolarization, lower expression of synaptopodin and nephrin and higher BSA permeability in podocytes exposed to HG and pretreated with t-BHQ $(p<0.05)$. These data suggested that the protection provided by activation of the Nrf2-ARE pathway in podocytes exposed to HG was partly dependent on Sirt1(Fig. 3 and 4).

Sirt1 and Nrf2 expression in $\mathrm{db} / \mathrm{db}$ mice

Obvious symptoms of diabetes, such as weight gain and increased appetite, water intake, urine and lethargy were observed in $\mathrm{db} / \mathrm{db}$ mice at approximately 8-10 weeks of age. As expected, the mean blood glucose levels in 8-week old db/db mice were notably high which

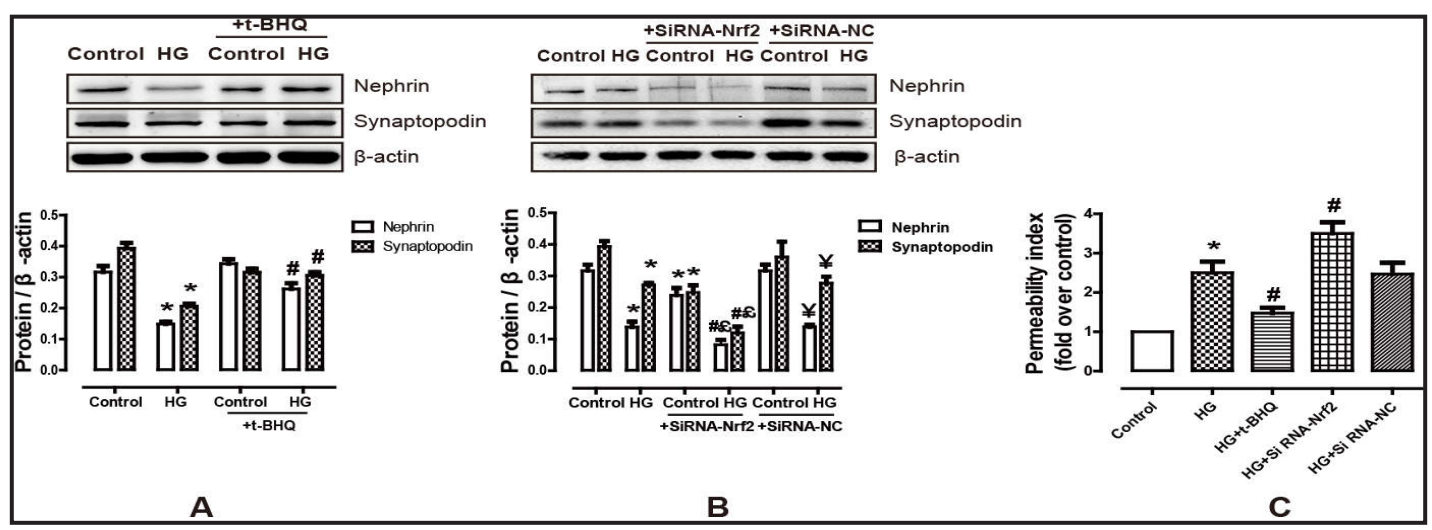

Fig. 1. Regulation of NRF2 can affect HG-induced human podocyte injury. A and B:Comparison of the expression of synaptopodin and nephrin in human podocytes from different groups. C: The BSA Permeability in podocytes from different groups. T-BHQ(20umol/l) were pretreated with for 4 hours, and then incubated with normal glucose $(5 \mathrm{mmol} / \mathrm{l})$ or high glucose $(30 \mathrm{mmol} / \mathrm{l})$ for 72 hours, respectly. Nrf2 were knockdowned by SiRNAs(SI RNA-Nrf2) and then these podocytes were exposed to normal glucose(5mmol/l) or high glucose (30mmol/l) for 72 hours, respectly. A disordered sequence SiRNAs(SI RNA-NC) was transfected into human podocytes and then these podocytes were exposed to normal glucose $(5 \mathrm{mmol} / \mathrm{l})$ or high glucose(30mmol/l) for 72 hours, respectly. ${ }^{*} \mathrm{p}<0.05$ vs Control group, $\# \mathrm{p}<0.05$ vs HG group, $€ \mathrm{p}<0.05$ vs Control+Si RNA-Nrf2 group, $¥ \mathrm{p}<0.05$ vs Control+Si RNA-NC group, $\mathrm{n}=3$. 


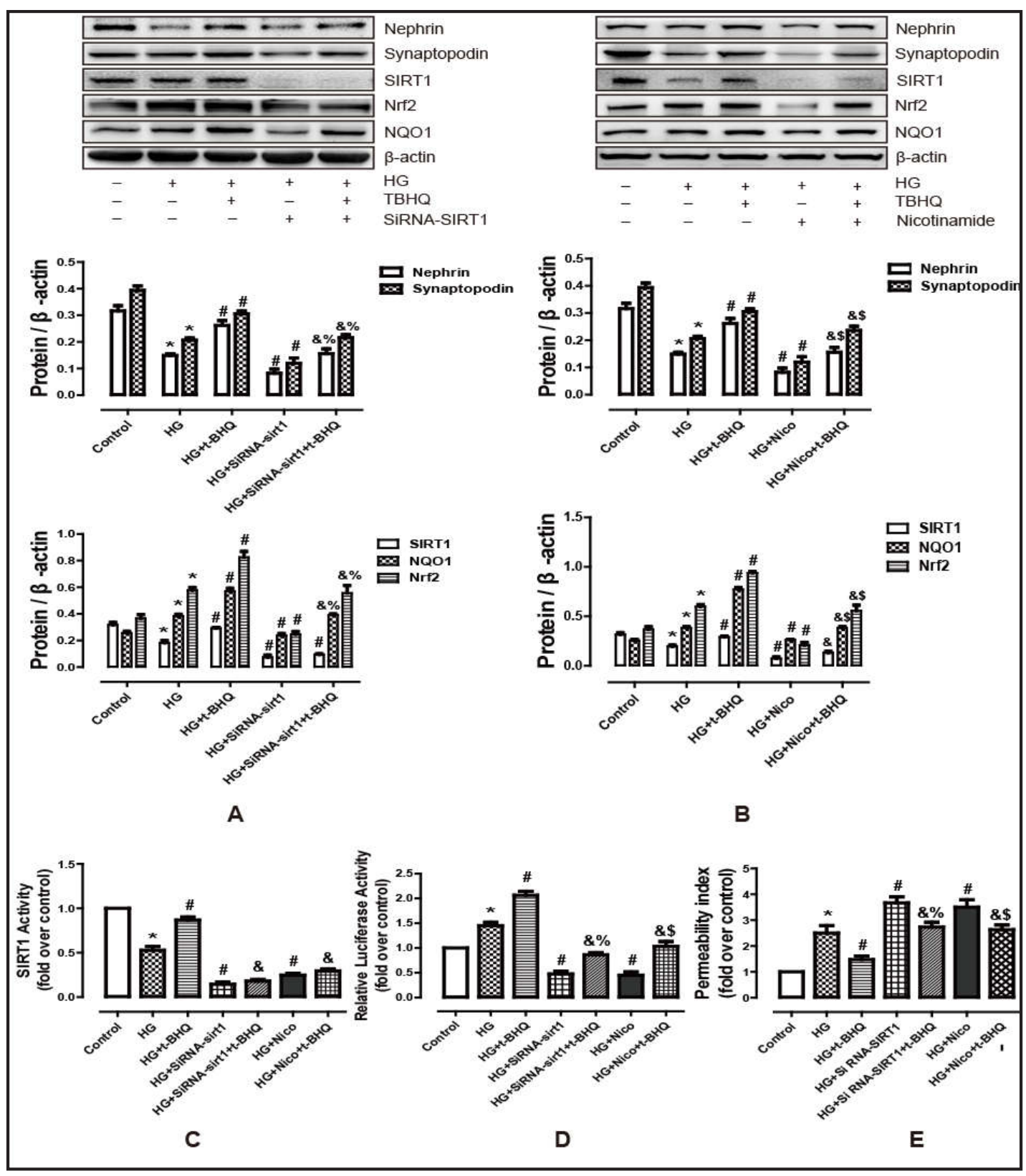

Fig. 2. Comparison of the activity of Nrf2-ARE pathway and SIRT1 in human podocytes from different groups. A and D: Comparison of the expression of SIRT1, Nrf2 and NQ01 proteins in podocytes from different groups. B and E: Comparison of the SIRT1 activity in human podocytes from different groups. $\mathrm{C}$ and F: Comparison of the Nrf2 transcriptional activity in human podocytes from different groups. T-BHQ(20umol/l) were pretreated with for 4 hours, and then incubated with normal glucose(5mmol/l) or high glucose $(30 \mathrm{mmol} / \mathrm{l})$ for 72 hours, respectly. Nrf2 were knock-downed by SiRNAs(SI RNA-Nrf2) and then these podocytes were exposed to normal glucose $(5 \mathrm{mmol} / \mathrm{l})$ or high glucose(30mmol/l) for 72 hours, respectly. A disordered sequence SiRNAs(SI RNA-NC) was transfected into human podocytes and then these podocytes were exposed to normal glucose $(5 \mathrm{mmol} / \mathrm{l})$ or high glucose $(30 \mathrm{mmol} / \mathrm{l})$ for 72 hours, respectly. ${ }^{*} \mathrm{p}<0.05$ vs Control group, \#p<0.05 vs HG group, $€ \mathrm{p}<0.05$ vs Control+Si RNA-Nrf2 group, ¥p<0.05 vs Control+Si RNA-NC group, $n=3$.

was consistently increased throughout the study period, compared with the $\mathrm{db} / \mathrm{m}$ mice. At the $24^{\text {th }}$ week, the mice were euthanatized and the renal cortex was collected for western

\section{KARGER}




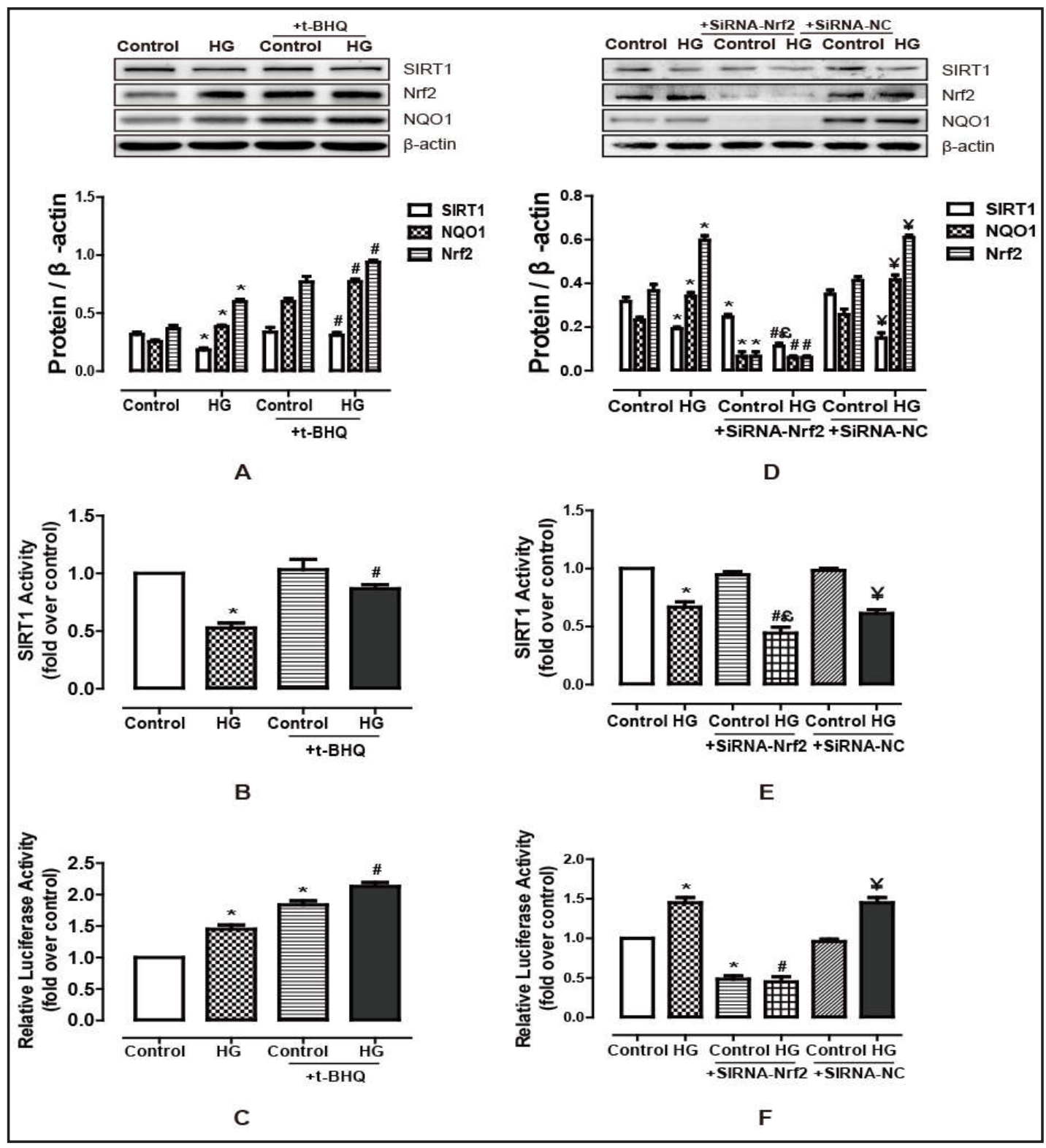

Fig. 3. Comparison of the effects of downregulated SIRT1 on damages in human podocytes induced by HG and Nrf2-ARE pathway. A and B: Comparison of the expression of Nephrin, Synaptopodin, SIRT1, Nrf2 and NQ01 proteins in podocytes from different groups. C: Comparison of the SIRT1 activity in human podocytes from different groups. D: Comparison of the Nrf2 transcriptional activity in human podocytes from different groups. E: Comparison of the BSA permeability in human podocytes from different groups. T-BHQ(20umol/l) were pretreated with for 4 hours, and then incubated with normal glucose(5mmol/l) or high glucose(30mmol/l) for 72 hours, respectly. Nicotinamide (NICO, 20nmol/l) were pretreated for 2 hours(+/- 20umol/l t-BHQ for 4 hours), and then incubated with high glucose(30mmol/l) for 72 hours. With the pretreatment of t-BHQ(20umol/l) for hours, SIRT1 were knock-downed by SiRNAs(SI RNA-SIRT1) and then these podocytes were exposed to high glucose( $30 \mathrm{mmol} / \mathrm{l})$ for 72 hours. ${ }^{*} \mathrm{p}<0.05$ vs Control group, $\# \mathrm{p}<0.05$ vs HG group, \&p<0.05 vs $\mathrm{HG}+\mathrm{t}-\mathrm{BHQ}$ group, \%p<0.05 vs $\mathrm{HG}+\operatorname{SiRNA}-\mathrm{SIRT} 1$ group, $\$ \mathrm{p}<0.05$ vs $\mathrm{HG}+$ Nicotinamide group, $\mathrm{n}=3$.

blot analysis. The 8-week old $\mathrm{db} / \mathrm{db}$ diabetic mice also exhibited significantly higher blood glucose levels, normal control animalsSirt1 expression was lower, while Nrf2 and NQO1 expression were higher in $\mathrm{db} / \mathrm{db}$ mice compared to $\mathrm{db} / \mathrm{m}$ mice. At the same time, nephrin 


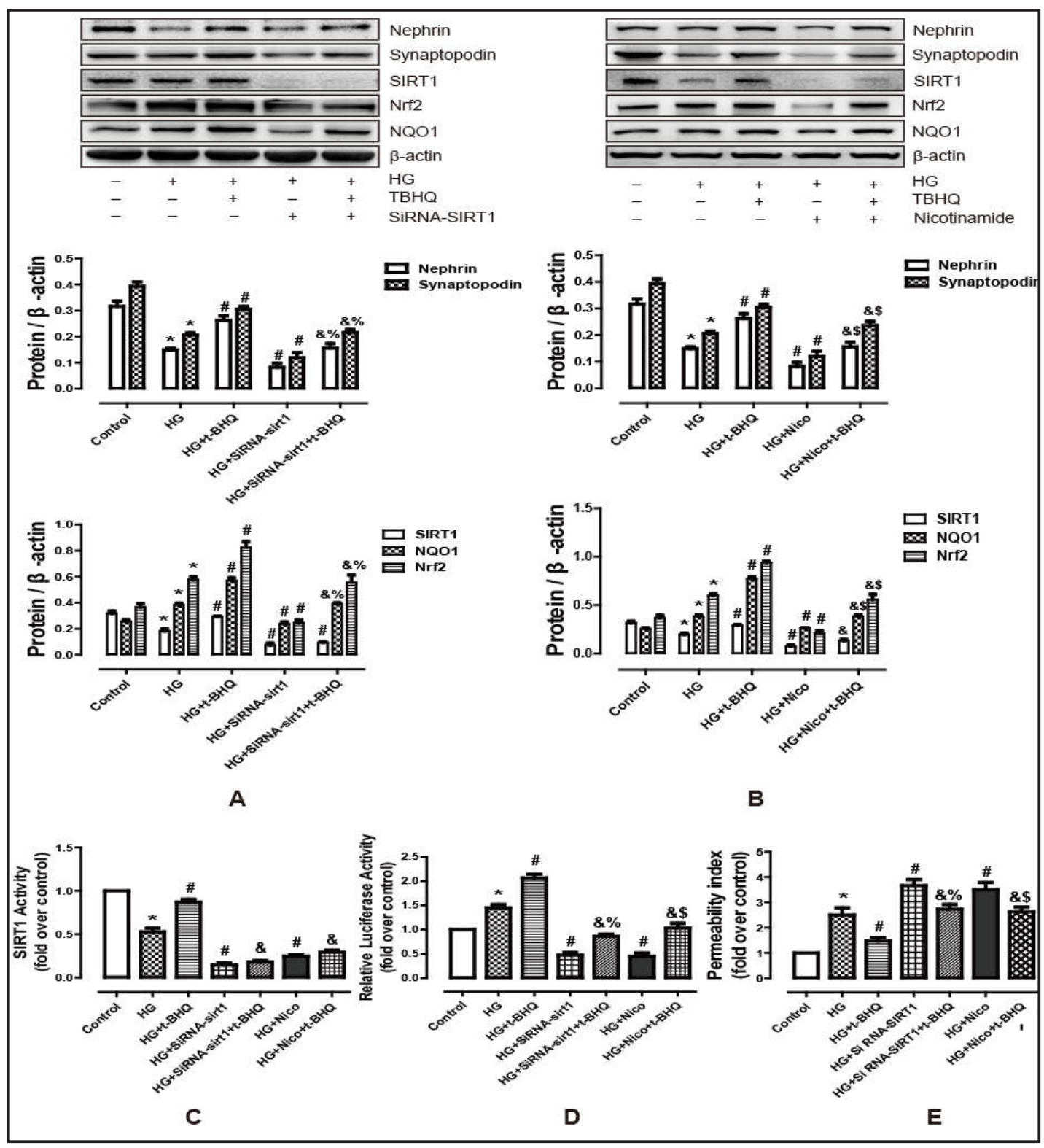

Fig. 4. Comparison of the levle of ATP, mitochondrial superoxide and membrane potential from different groups. A: Comparison of the levle of ATP from different groups. B: Comparison of mitochondrial superoxide and membrane potential from different groups. T-BHQ(20umol/l) were pretreated with for 4 hours, and then incubated with normal glucose $(5 \mathrm{mmol} / \mathrm{l})$ or high glucose $(30 \mathrm{mmol} / \mathrm{l})$ for 72 hours, respectly. Nicotinamide (NICO, 20nmol/l) were pretreated for 2 hours(+/- 20umol/l t-BHQ for 4 hours), and then incubated with high glucose $(30 \mathrm{mmol} / \mathrm{l})$ for 72 hours. Nrf2 were knock-downed by SiRNAs(SI RNA-Nrf2) and then these podocytes were exposed to normal glucose $(5 \mathrm{mmol} / \mathrm{l})$ or high glucose(30mmol/l) for 72 hours, respectly. A disordered sequence SiRNAs(SI RNA-NC) was transfected into human podocytes and then these podocytes were exposed to normal glucose $(5 \mathrm{mmol} / \mathrm{l})$ or high glucose $(30 \mathrm{mmol} / \mathrm{l})$ for 72 hours, respectly. With the pretreatment of t-BHQ(20umol/l) for hours, SIRT1 were knock-downed by SiRNAs(SI RNA-SIRT1) and then these podocytes were exposed to high glucose (30mmol/l) for 72 hours. ${ }^{*}$ p $<0.05$ vs Control group, \#p<0.05 vs HG group, \&p<0.05 vs HG+t-BHQ group, \%p<0.05 vs HG+SiRNA-SIRT1 group, $\$ \mathrm{p}<0.05$ vs $\mathrm{HG}+$ Nicotinamide group, $\mathrm{n}=3$.

and synaptopodin expression in the renal cortex was lower in $\mathrm{db} / \mathrm{db}$ mice than in $\mathrm{db} / \mathrm{m}$ mice, and more severe mesangial matrix deposition, collagen deposition and mitochondrial 
structural damage was found in podocytes from $\mathrm{db} / \mathrm{db}$ compared to $\mathrm{db} / \mathrm{m}$ mice (Fig. 5, 6 and 7).

Changes in pathology, physiology and mitochondrial injuries in $\mathrm{db} / \mathrm{db}$ mice induced by $t-B H Q$

To explore the effect of Nrf2-ARE pathway activation on pathology, physiology and mitochondrial injuries in $\mathrm{db} / \mathrm{db}$ mice, $\mathrm{t}-\mathrm{BHQ}$ was dissolved in the double-distilled drinking water of $\mathrm{db} / \mathrm{db}$ and $\mathrm{db} / \mathrm{m}$ mice. Nrf2 and NQO1 expression in the renal cortex was increased in both $\mathrm{db} / \mathrm{db}$ and $\mathrm{db} / \mathrm{m}$ mice drinking t-BHQ-laced water, which indicated that the Nrf2-ARE pathway was activated by t-BHQ in these mice. Furthermore, urinary albumin/creatinine was decreased and nephrin and synaptopodin expression was increased in the renal cortex of db/ $\mathrm{db}$ mice drinking t-BHQ water compared with $\mathrm{db} / \mathrm{db}$ mice drinking double-distilled water alone. At the same time, the more severe mesangial matrix deposition, collagen deposition and mitochondrial structural damage in podocytes from $\mathrm{db} / \mathrm{db}$ mice were alleviated when the mice drank t-BHQ-laced water (Fig. 5, 6 and 7).

Changes in pathology, physiology and mitochondrial injuries in $\mathrm{db} / \mathrm{db}$ mice induced by nicotinamide

Sirt1 expression in the renal cortex was decreased in $\mathrm{db} / \mathrm{db}$ mice compared to $\mathrm{db} / \mathrm{m}$ mice. When we gave nicotinamide by gavage to inhibit Sirt1 activity in these mice, we found that urinary albumin/creatinine was increased and nephrin and synaptopodin expression was decreased, while more severe mesangial matrix deposition, collagen deposition and mitochondrial structural damage occurred in podocytes from $\mathrm{db} / \mathrm{db}$ mice treated with nicotinamide than in mice without nicotinamide treatment (Fig. 5, 6 and 7).

Changes in pathology, physiology and mitochondrial injuries induced by $t-B H Q$ in $d b / d b$ mice with and without nicotinamide treatment

When we gave nicotinamide and t-BHQ to $\mathrm{db} / \mathrm{db}$ mice, we found higher urinary albumin/ creatinine and lower nephrin and synaptopodin expression, with more severe mesangial matrix deposition and collagen deposition on pathological slides, and greater mitochondrial structural damage in podocytes compared to $\mathrm{db} / \mathrm{db}$ mice treated only with t-BHQ. Moreover, there was lower urinary albumin/creatinine, higher nephrin and synaptopodin expression, diminished mesangial matrix and collagen deposition and less mitochondrial structural damage in podocytes from $\mathrm{db} / \mathrm{db}$ mice treated with both t-BHQ and nicotinamide than in untreated $\mathrm{db} / \mathrm{db}$ mice, which suggested that the protection provided by $\mathrm{tBHQ}$ activation of the Nrf2-ARE pathway in db/db mice was partly dependent on Sirt1 (Fig. 5, 6 and 7).

Fig. 5. Physical and metabolic parameters of the animals. A. Urinary albumin to creatinine ratio. B. Blood glucose. C. Serum urea nitrogen. D. Left kidney weight to body weight ratio. ${ }^{*} \mathrm{P}<0.05$ vs. $\mathrm{db} / \mathrm{m}$ mice, $\# \mathrm{P}<0.05$ vs. $\mathrm{db} / \mathrm{db}$ mice, $\& \mathrm{p}<0.05$ vs $\mathrm{db} / \mathrm{db}$ mice +t-BHQ group, $\$ \mathrm{p}<0.05 \mathrm{vs} \mathrm{db} / \mathrm{db}$ mice+Nicotinamide group, $\mathrm{n}=6$.

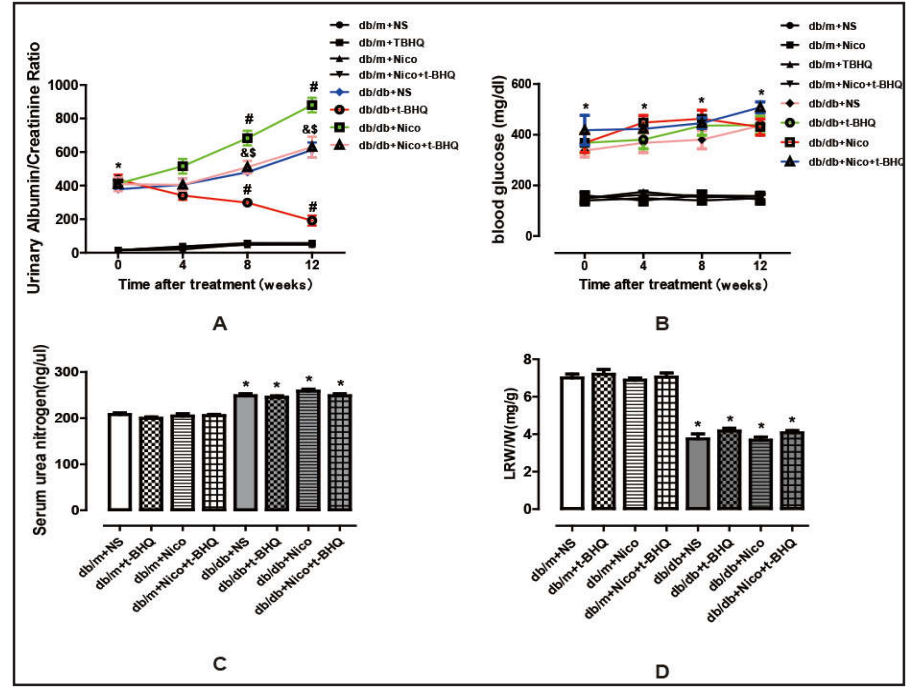




\section{$\begin{array}{lll}\text { Cellular Physiology } & \text { Cell Physiol Biochem 2018;48:1-15 } \\ \text { DOI: 10.1159/000491658 } & 0 \text { 2018 The Author(s). Published by S. Karger AG, Basel }\end{array}$

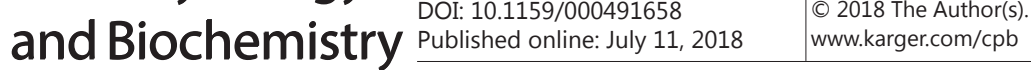

\section{Discussion}

The present study has demonstrated a protective effect of the Nrf2-ARE pathway and exposed its molecular mechanism in podocytes under hyperglycemic condition and also in $\mathrm{db} / \mathrm{db}$ diabetic mice. Human podocyte damage induced by hyperglycemia was attenuated by t-BHQ (an activator of the Nrf2-ARE pathway), while the protection afforded by t-BHQ and Nrf2-ARE pathway expression was partially attenuated when Sirt1 expression or activity was inhibited. At the same time, when Sirt1 expression was decreased, Nrf2 expression was also inhibited, while Sirt1 expression was increased by the activation of Nrf2-ARE

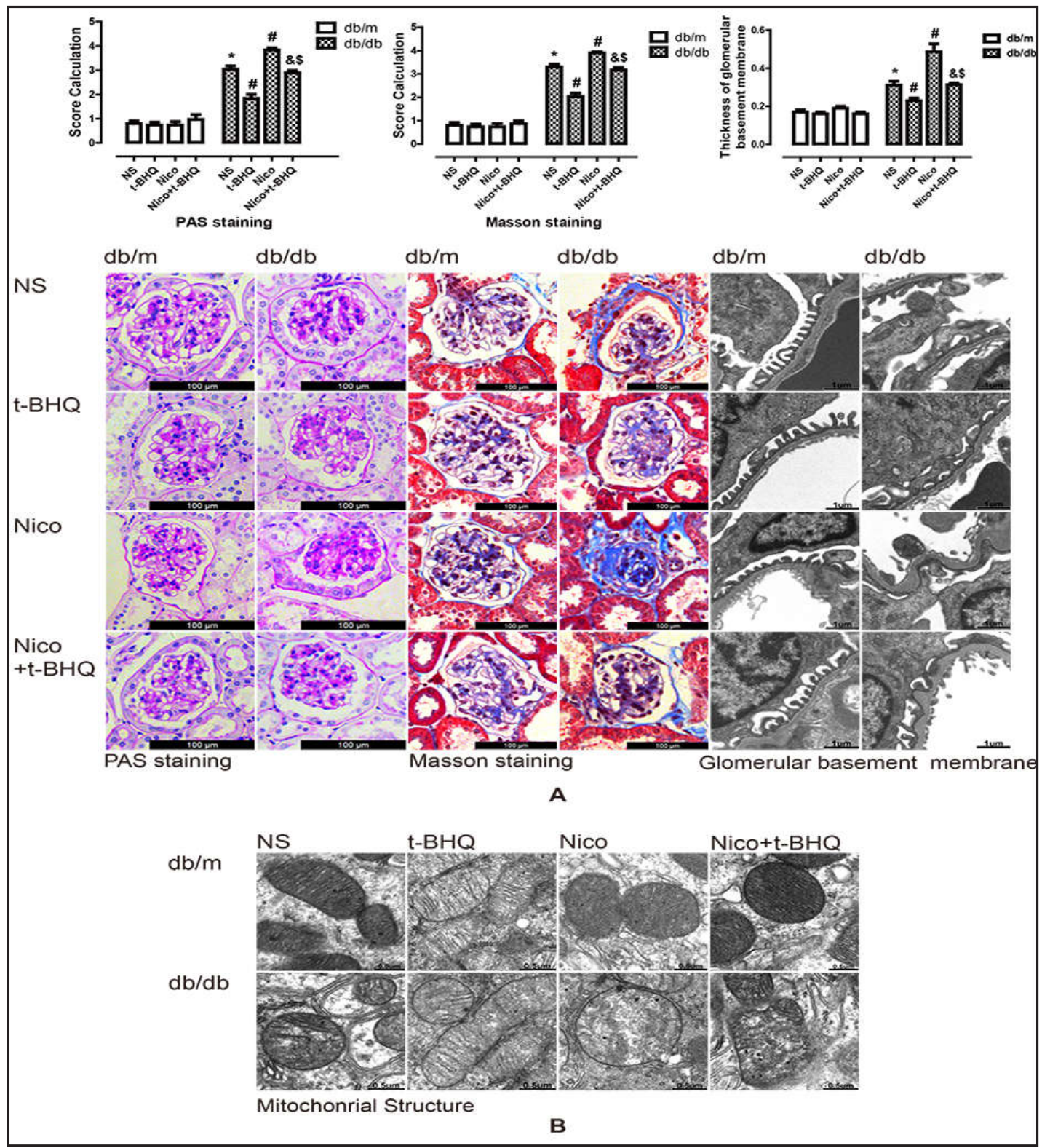

Fig. 6. Nicotinamde and t-BHQ affected pathology in $\mathrm{db} / \mathrm{db}$ mice. A: Score calculation of HE, Masson staining and calculation of thickness of glomerular basement membrane. B: Mitochondrial structure image. PAS and Masson staining: bar=100um, electron microscopy to image the mitochondrial structure: bar=1um, electron microscopy to assess glomerular basement membrane: bar $=0.5 \mathrm{um}$. $* \mathrm{P}<0.05 \mathrm{vs}$. db $/ \mathrm{m}$ mice, $\# \mathrm{P}<0.05 \mathrm{vs}$. db/ db mice, $\&$ p < 0.05 vs db/db mice +t-BHQ group, $\$ \mathrm{p}<0.05$ vs db/db mice+Nicotinamide group, $\mathrm{n}=6$.

\section{KARGER}




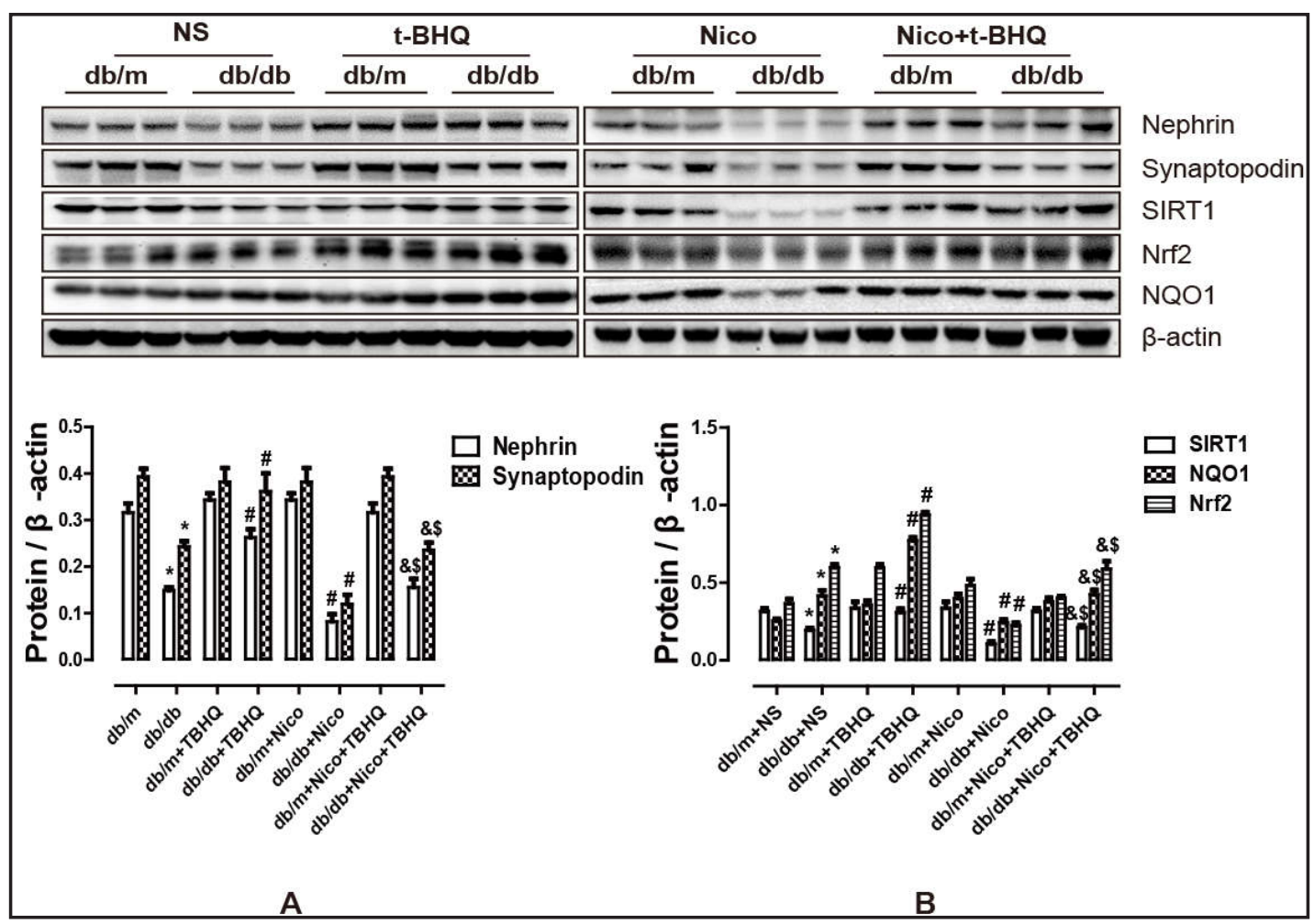

Fig. 7. Nicotinamde inhibits Nrf2-ARE pathway in mice with diabetic nephropathy. A: Comparision of expression of Nephrin and Synaptopodin proteins in podocytes from different groups. B: Comparision of protein expression of SIRT1, Nrf2 and NQ01 in podocytes from different groups. ${ }^{*} \mathrm{P}<0.05 \mathrm{vs}$. db/m mice $\# \mathrm{P}<0.05$ vs. $\mathrm{db} / \mathrm{db}$ mice, $\& \mathrm{p}<0.05$ vs $\mathrm{db} / \mathrm{db}$ mice $+\mathrm{t}-\mathrm{BHQ}$ group, $\$ \mathrm{p}<0.05 \mathrm{vs} \mathrm{db} / \mathrm{db}$ mice + Nicotinamide group, $\mathrm{n}=6$.

pathway. When we gave nicotinamide and t-BHQ to $\mathrm{db} / \mathrm{db}$ mice, we observed higher urinary albumin/creatinine and lower nephrin and synaptopodin expression, along with more severe mesangial matrix deposition, collagen deposition on pathological slides and greater mitochondrial structural damage in podocytes compared to $\mathrm{db} / \mathrm{db}$ mice treated with tBHQ alone. Taken together, these data suggest that some potential relationship between Sirt1 and the Nrf2-ARE anti-oxidative pathway that may provide positive effects on hyperglycemia induced podocyte injuries and kidney injuries, besides, the protection provided by tBHQinduced activation of the Nrf2-ARE pathway in $\mathrm{db} / \mathrm{db}$ mice is partly dependent on Sirt1.

Previously, we have shown that the Nrf-ARE pathway protects mice podocytes exposed to HG [20], but the underlying molecular mechanism remains unknown. Here, we found that mitochondrial dysfunction and podocyte damage were induced by HG. However, when these podocytes were treated by t-BHQ, the Nrf2-ARE pathway was activated and mitochondrial dysfunction and podocyte damage were attenuated, whereas more severe mitochondrial dysfunction and podocyte damage were induced by HG when the podocytes were transfected by Nrf2 siRNAs, which establishes a role for the Nrf2-ARE pathway in protecting against mitochondrial damage in human podocytes. The next step was to explore the underlying mechanism. We discovered some potential relationship between Sirt1 and the Nrf2-ARE pathway in podocytes, and the protection provided by Nrf2-ARE pathway activation was attenuated in human podocytes and in $\mathrm{db} / \mathrm{db}$ mice when Sirt1 expression or activity was inhibited. All of these data suggest that the protection provided by Nrf2-ARE activation in podocytes occurred partly through the Sirt1 pathway, which may be a promising strategy for the prevention of podocyte loss in the treatment glomerular diseases.

$\mathrm{Nrf} 2$ is a transcription factor that regulates coordinated key antioxidant responses in cells, and its activation can exert protective effects in a wide variety of animal models KARGER 


\section{Cellular Physiology Cell Physiol Biochem 2018;48:1-15

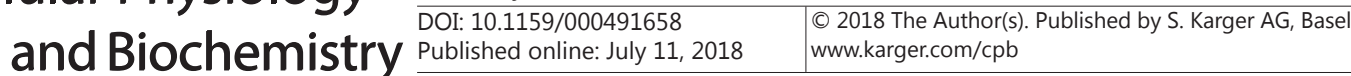 \\ Zhang et al.: Nrf2-ARE Pathway in Mitochondrial Dysfunction}

of oxidative stress-related injury and inflammatory disease [27]. Nrf2 activation with sulforaphane suppressed nephropathy and significantly improved metabolic indices associated with type 2 diabetes mellitus in a streptozocin-induced mouse diabetes model [28]. Similar findings with other known activators such as resveratrol support the therapeutic targeting of this system to ameliorate the oxidative damage and glucose-induced mesangial cell proliferation, inflammation, and fibrosis which underlies diabetic nephropathy [29, 30$]$. Lower levels of Nrf2 in the peripheral blood of patients with chronic DKD further support the contribution of an impaired Nrf2 antioxidant signaling pathway to systemic oxidative overload and inflammation in diabetic nephropathy [31]. Few studies have focused on podocytes, particularly podocyte mitochondrial function, and to the best of our knowledge, we are the first to report the protection provided by Nrf2-ARE pathway activation against mitochondrial dysfunction in podocytes induced by HG.

Sirt1 is a member of a family of proteins with homology to the Silence Information Regulator 2 (SIR2) gene of yeast S. cerevisiae and plays an important role in the regulation of cell death/ survival and stress responses in mammals [32.] Although primarily a nuclear protein, the deacetylation of Sirt1 by peroxisome proliferator-activated receptor gamma Coactivator$1 \alpha$, has been extensively implicated in metabolic control and mitochondrial biogenesis and was proposed to partially underlie Sirt1's role in caloric restriction and its impacts on cell senescence [33]. Other experiments studied in advanced glycation-end products (AGEs) treated glomerular mesangial cells (GMCs), crosstalk between Sirt1 and Keap1/Nrf2/ARE anti-oxidative pathway forms a positive feedback loop to inhibit the protein expressions of FN and TGF- $\beta 1$ [23]. In our experiments, we found that Sirt1 expression and activity were inhibited by HG, and mitochondrial dysfunction and podocyte damage were more severe when we lowered Sirt1 in podocytes exposed to HG. Interestingly, Nrf2 also positively regulated Sirt1 at the protein expression and deacetylase activity levels as evidenced by t-BHQ and specific siRNA targeting Nrf2, while the protective effects of t-BHQ against mitochondrial dysfunction and podocyte damage exposed to HG was partly attenuated when we inhibited Sirt1 with siRNA. These results suggest that the protection provided by activation of the Nrf2-ARE pathway by tBHQ in db/db mice is partially dependent on Sirt1.

There are some limitations that may affect the conclusions that can be drawn from this study. First, we used the db/db type 2 diabetic mouse model and t-BHQ as an activator of Nrf2 to explore the role of the Nrf2-ARE pathway in mitochondrial damage in podocytes, and thus could not rule out non-specific effects of t-BHQ. Future studies should use podocytes drawn from specific Nrf2 knockout mice to avoid non-specific effects. Second, mice were given t-BHQ in their drinking water, so the final concentration of the drug may have varied in mice depending of the volume of water consumed. Third, nicotinamide and t-BHQ were given to the same mice and we could not rule out chemical cross-reactions in these mice although no obvious side effects were observed. Besides, the potential connection between Sirt1 and Nrf2-ARE pathway are not fully understood, further research is needed to study the internal relation and mechanism and.

In conclusion, our results indicate that crosstalk between Sirt1 and the Nrf2-ARE antioxidative pathway may forms a positive feedback loop and that the protection provided by the tBHQ-activated Nrf2-ARE pathway in all these data illustrates $\mathrm{db} / \mathrm{db}$ mice is partly dependent on Sirt1.

\section{Acknowledgements}

This work was supported by a grant from guangzhou science and technolody project (201510010152). The funders had no role in study design, data collection and analysis, decision to publish, or preparation of the manuscript.

\section{Disclosure Statement}

The authors declare no competing financial interests.

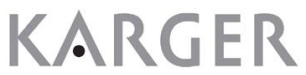




\section{Cellular Physiology Cell Physiol Biochem 2018;48:1-15 \begin{tabular}{l|l} 
and Biochemistry Published 10.1159/000491658 & $\begin{array}{l}\text { (c) } 2018 \text { The Author(s). Published by S. Karger AG, Basel } \\
\text { www.karger.com/cpb }\end{array}$
\end{tabular} \\ Zhang et al.: Nrf2-ARE Pathway in Mitochondrial Dysfunction}

\section{References}

1 Breyer MD, Susztak K: The next generation of therapeutics for chronic kidney disease. Nat Rev Drug Discov 2016;15:568-588.

- Khan SS, Quaggin SE: Therapies on the horizon for diabetic kidney disease. Curr Diab Rep DOI: 10.1007/ s11892-015-0685-3.

3 Brownlee M: The pathobiology of diabetic complications: a unifying mechanism. Diabetes 2005;54:16151625.

-4 Nishikawa T, Edelstein D, Du XL, Yamagishi S, Matsumura T, Kaneda Y, Yorek MA, Beebe D, Oates PJ, Hammes HP, Giardino I, Brownlee M: Normalizing mitochondrial superoxide production blocks three pathways of hyperglycaemic damage. Nature 2000;404:787-790.

5 Yang S, Zhang J, Wang S, Shi J, Zhao X: Knockdown of Angiopoietin-Like Protein 2 Ameliorates Diabetic Nephropathy by Inhibiting TLR4. Cell Physiol Biochem 2017;43:685-696.

-6 Lin JS, Susztak K: Podocytes: the weakest link in diabetic kidney disease? Curr Diab Rep D0I: 10.1007/ s11892-016-0735-5.

7 Guo J, Lu C, Zhang F, Yu H, Zhou M, He M, Wang C, Zhao Z, Liu Z: VDR Activation Reduces Proteinuria and High-Glucose-Induced Injury of Kidneys and Podocytes by Regulating Wnt Signaling Pathway. Cell Physiol Biochem 2017;43:39-51.

8 Coimbra TM, Janssen U, Gröne HJ, Ostendorf T, Kunter U, Schmidt H, Brabant G, Floege J: Early events leading to renal injury in obese Zucker rats with type II diabetes. Kidney Int 2000;57:167-182.

-9 Menini S, Iacobini C, Oddi G, Ricci C, Simonelli P, Fallucca S, Grattarola M, Pugliese F, Pesce C, Pugliese G: Increased glomerular apoptosis in rats with streptozotocin-induced diabetes mellitus: role in the development of diabetic glomerular disease. Diabetologia 2007;50:2591-2599.

10 Li S, Liu X, Lei J, Yang J, Tian P, Gao Y: Crocin Protects Podocytes Against Oxidative Stress and Inflammation Induced by High Glucose Through Inhibition of NF- $\kappa$ B. Cell Physiol Biochem 2017;42:1481-1492.

-11 Toyoda M, Najafian B, Kim Y, Caramori ML, Mauer M: Podocyte detachment and reduced glomerular capillary endothelial fenestration in human type 1 diabetic nephropathy. Diabetes 2007;56:2155-2160.

12 Weil EJ, Lemley KV, Mason CC, Yee B, Jones LI, Blouch K, Lovato T, Richardson M, Myers BD, Nelson RG: Podocyte detachment and reduced glomerular capillary endothelial fenestration promote kidney disease in type 2 diabetic nephropathy. Kidney Int 2012;82:1010-1017.

13 Picard M, Taivassalo T, Gouspillou G, Hepple RT: Mitochondria: isolation, structure and function. J Physiol 2011;589:4413-4421.

14 Takahashi N, Kimura H, Mizuno S, Hara M, Hirayama Y, Kurosawa H, Tsutaya S, Yasujima M, Yokoi S, Mikami D, Kasuno K, Naiki H, Iwano M, Yoshida H: Severe intraglomerular detachment of podocytes in a Gitelman syndrome patient. Clin exper nephrol 2012;16:495-500.

15 Lei CT, Wei YH, Tang H, Wen Q, Ye C, Zhang C, Su H: PKC- $\alpha$ Triggers EGFR Ubiquitination, Endocytosis and ERK Activation in Podocytes Stimulated with High Glucose. Cell Physiol Biochem 2017;42:281-294.

16 Su M, Dhoopun AR, Yuan Y, Huang S, Zhu C, Ding G, Liu B, Yang T, Zhang A: Mitochondrial dysfunction is an early event in aldosterone-induced podocyte injury. Am J Physiol Renal Physiol 2013;305:F520-F531.

17 Zhao M, Yuan Y, Bai M, Ding G, Jia Z, Huang S, Zhang A: PGC-1 $\alpha$ overexpression protects against aldosteroneinduced podocyte depletion: role of mitochondria. Oncotarget 2016;7:12150-12162.

18 Huang H, You Y, Lin X, Tang C, Gu X, Huang M, Qin Y, Tan J, Huang F: Inhibition of TRPC6 Signal Pathway Alleviates Podocyte Injury Induced by TGF- $\beta 1$. Cell Physiol Biochem 2017;41:163-172.

19 Keum YS, Choi BY: Molecular and chemical regulation of the Keap1-Nrf2 signaling pathway. Molecules 2014;19:10074-10089.

20 Wang C, Li C, Peng H, Ye Z, Zhang J, Liu X, Lou T: Activation of the Nrf2-ARE pathway attenuates hyperglycemiamediated injuries in mouse podocytes. Cell Physiol Biochem 2014;34:891-902.

21 Kang H, Oka S, Lee DY, Park J, Aponte AM, Jung YS, Bitterman J, Zhai P, He Y, Kooshapur H, Ghirlando R, Tjandra N, Lee SB, Kim MK, Sadoshima J, Chung JH: Sirt1 carboxyl-domain is an ATP-repressible domain that is transferrable to other proteins. Nat Commun DOI: $10.1038 /$ ncomms 15560.

-22 Sulaiman M, Matta MJ, Sunderesan NR, Gupta MP, Periasamy M, Gupta M: Resveratrol, an activator of SIRT1, upregulates sarcoplasmic calcium ATPase and improves cardiac function in diabetic cardiomyopathy. Am J Physiol Heart Circ Physiol 2010;298:H833-H843. 


\section{Cellular Physiology Cell Physiol Biochem 2018;48:1-15 \begin{tabular}{l|l} 
DOI: 10.1159/000491658 & $\begin{array}{l}\text { O 2018 The Author(s). Published by S. Karger AG, Basel } \\
\text { www.karger.com/cpb }\end{array}$
\end{tabular} \\ Zhang et al.: Nrf2-ARE Pathway in Mitochondrial Dysfunction}

23 Huang K, Gao X, Wei W: The crosstalk between Sirt1 and Keap1/Nrf2/ARE anti-oxidative pathway forms a positive feedback loop to inhibit FN and TGF- $\beta 1$ expressions in rat glomerular mesangial cells. Exp Cell Res 2017;361:63-72.

-24 Saleem MA, O’Hare MJ, Reiser J, Coward RJ, Inward CD, Farren T, Xing CY, Ni L, Mathieson PW, Mundel P: A conditionally immortalized human podocyte cell line demonstrating nephrin and podocin expression. J Am Soc Nephrol 2002;13:630-638.

25 Wang S, Zhang C, Zhang M, Liang B, Zhu H, Lee J, Viollet B, Xia L, Zhang Y, Zou MH: Activation of AMP-activated protein kinase alpha2 by nicotine instigates formation of abdominal aortic aneurysms in mice in vivo. Nat Med 2012;18:902-910.

26 Qi H, Casalena G, Shi S, Yu L, Ebefors K, Sun Y, Zhang W, D’Agati V, Schlondorff D, Haraldsson B, Bottinger E, Daehn L: Glomerular Endothelial Mitochondrial Dysfunction Is Essential and Characteristic of Diabetic Kidney Disease Susceptibility. Diabetes 2017; 66: 763-778.

27 Nguyen T, Nioi P, Pickett CB: The Nrf2-antioxidant response element signaling pathway and its activation by oxidative stress. J Biol Chem 2009;284:13291-13295.

28 Zheng H, Whitman SA, Wu W, Wondrak GT, Wong PK, Fang D, Zhang DD: Therapeutic potential of Nrf2 activators in streptozotocin-induced diabetic nephropathy. Diabetes 2011;60:3055-3066.

29 Jiang B, Guo L, Li BY, Zhen JH, Song J, Peng T, Yang XD, Hu Z, Gao HQ: Resveratrol attenuates early diabetic nephropathy by down-regulating glutathione S-transferases mu in diabetic rats. J Med Food 2013;16:481-486.

-30 Cui W, Li B, Bai Y, Miao X, Chen Q, Sun W, Tan Y, Luo P, Zhang C, Zheng S, Epstein PN, Miao L, Cai L: Potential role for Nrf2 activation in the therapeutic effect of MG132 on diabetic nephropathy in OVE26 diabetic mice. Am J Physiol Endocrinol Metab 2013; 304:E87-99.

-31 Pedruzzi LM, Cardozo LF, Daleprane JB, Stockler-Pinto MB, Monteiro EB, Leite M Jr, Vaziri ND, Mafra D: Systemic inflammation and oxidative stress in hemodialysis patients are associated with down-regulation of Nrf2. J Nephrol 2015;28:495-501.

32 Longo VD, Kennedy BK: Sirtuins in aging and age-related disease. Cell 2006;126:257-268.

33 Tang BL: Sirt1 and the Mitochondria. Mol Cells 2016;39:87-95. 